\title{
Structural results for partially nested LQG systems over graphs
}

\author{
Ashutosh Nayyar ${ }^{1} \quad$ Laurent Lessard ${ }^{2}$ \\ American Control Conference, pp. 5457-5464, 2015
}

\begin{abstract}
We identify a broad class of decentralized outputfeedback LQG systems for which the optimal control strategies have a simple and intuitive estimation structure. We consider cases for which the coupling of dynamics among subsystems and the inter-controller communication are characterized by the same directed graph. For the class of graphs known as multitrees, we show that each controller need only estimate the states of the subsystems it affects (its descendants) as well as the subsystems it observes (its ancestors). The optimal control action for each controller is a linear function of the estimate it computes and the estimates computed by its ancestors. Moreover, all state estimates may be updated recursively, much like a Kalman filter.
\end{abstract}

\section{Introduction}

The past decade has seen a resurgence of interest in decentralized control problems; where different controllers must make decisions using different subsets of the available information. A central question in the investigation of decentralized problems is whether the ever-growing information history available to controllers be aggregated without compromising performance. In other words: do the optimal controllers have convenient sufficient statistics? In this paper, we identify a broad class of information structures and associated control problems for which the above questions have an affirmative answer.

We represent information structures by using directed acyclic graphs (DAG), where each node represents both a subsystem and its associated controller, and the edges indicate both the influence of state dynamics between subsystems as well as information-sharing between controllers. An example is shown in Figure 1.

Our main result, formally stated in Section 3, may be summarized by the following intuitive statement: each controller must maintain state estimates of the subsystems that it observes (its ancestors) as well as state estimates of subsystems that it affects through its deci-

\footnotetext{
${ }^{1}$ A. Nayyar is with the Ming Hsieh Department of Electrical Engineering, University of Southern California, Los Angeles, CA 90089, USA. ashutosn@usc.edu. A. Nayyar was supported by NSF under grant number CNS-144690.

${ }^{2} \mathrm{~L}$. Lessard is with the Department of Mechanical Engineering at the University of California, Berkeley, CA 94720, USA. lessard@berkeley.edu. L. Lessard was supported by NASA under Grant No. NRA NNX12AM55A.
}

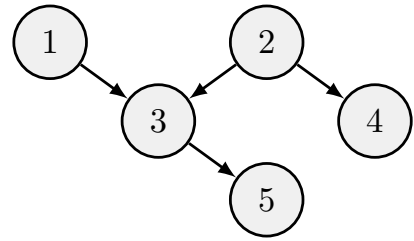

Figure 1: Directed acyclic graph (DAG) representing the information structure of a decentralized control problem. An edge $i \rightarrow j$ means that subsystem $i$ affects subsystem $j$ through its dynamics and controller $i$ shares its information with controller $j$ (but not vice versa).

sions (its descendants). In the example of Figure 1, the controller at node 1 must estimate the states of nodes $\{1,3,5\}$, while the controller at node 4 must estimate the states of nodes $\{2,4\}$. The optimal control action for each controller is a linear function of the estimate it computes as well as the estimates computed by all of its ancestors. In addition to proving the sufficient statistics, we show that they admit a recursive representation, similar to that of the Kalman filter.

Our results hold for LQG systems; all dynamics are linear (possibly time-varying), process noise and measurement noise is Gaussian, and the cost function is quadratic over a finite time horizon. We must also impose some restrictions on which pairs of nodes may be coupled by the quadratic cost and which pairs of nodes may be driven by correlated Gaussian disturbances. These assumptions are stated precisely in Section 2.4. The associated DAG may be any multitree. In other words: in the transitive reduction of the DAG, each pair of nodes can be connected by at most one directed path. For example, if we add the edge $4 \rightarrow 5$ to Figure 1 the multitree assumption is now violated because there are now two separate directed paths connecting 2 to 5 .

A key aspect of this work is that we consider output feedback. While the presence of measurement noise makes the problem considerably more difficult to solve than the state-feedback case, we nevertheless show that the optimal controller has a simple and intuitive structure.

Due to space constraints, we will provide a proof outline of our main result only for the specific information structure of Figure 1. Although this graph is relatively simple, it captures the salient features of our approach and it will be clear how the proof can be generalized to more general graphs. For the interested reader, a detailed proof of the general multitree case is available online [16]. 


\subsection{Prior work}

The analytical and computational difficulty of general decentralized control problems with arbitrary information structure has been widely acknowledged $[2,27]$. There has been considerable interest in identifying classes of information structures that may be "easier" to solve.

In the team-theory inspired literature on decentralized control, a key simplifying feature is the notion of partial nestedness (PN) [4]. A decentralized LQG problem with a PN information structure admits a linear control strategy that is globally optimal. Further, it can be reduced (at least for finite horizon problems) to a static LQG team problem for which person-by-person optimal strategies are globally optimal [19]. Despite these facts, a universal and computationally efficient methodology for finding optimal strategies for all partially nested problems remains elusive.

Another approach is to formulate the control problem as a closed-loop norm optimization. In this framework, some simplifying properties of the plant and information constraint have been identified. These properties imply convexity of the set of achievable closed-loop maps. Examples of such properties include quadratic invariance (QI) [20], funnel causality [1], and certain hierarchical architectures [18]. Despite the convexity, the optimization problem is infinite dimensional in general and therefore hard to solve.

A widely used model for PN/QI problems in decentralized control is to assume the plant dynamics and the controller structure are characterized by the same directed graph. The first solutions to problems of this kind assumed state feedback $[22,23]$. The case of noisy measurements (output feedback) was found to be considerably more difficult because the problem cannot be split into separate problems as in the state feedback case. However, the problem-splitting approach still holds if only the leaf-nodes have noisy measurements [5, 17, 24]. The first solution to a full output feedback case was for a twosubsystem problem $[12,13]$. This result was later generalized to star-shaped graphs in [11] and linear chain graphs in [25].

Although the above works considered continuous-time systems, the results can easily be adapted to a discretetime setting as well. Discrete-time formulations for decentralized problems were used in the 70's for treating partially nested "delayed-sharing" cases [7, 21, 26, 28]. More recently, this notion was generalized to delays characterized in terms of distance on a DAG $[8,9,10]$.

The focus of the above works is to find a state-space representation for the optimal decentralized control law; there is typically no immediate way of obtaining a meaningful interpretation of the optimal controller states. One notable exception is [14], where the controller states were interpreted as minimum mean-squared error (MMSE) estimates of the plant states.
An alternative approach is to use a team-theoretic perspective. The two-subsystem output feedback problem was solved in this manner in [15]. We use a similar approach in the present work to extend the output feedback results to a broader class of DAGs. The advantage of a team-theoretic approach is that structural results emerge naturally, and one can deduce the optimal controller's sufficient statistics without solving for gains explicitly. Indeed, the paper [15] derives structural results for a finite-horizon formulation with a linear time-varying plant, whereas the works $[11,12,13,14,25]$ address linear time-invariant plants and find the infinite-horizon steadystate optimal controller.

The paper is organized as follows. We cover notation and basic assumptions in Section 2, the main results are presented in Section 3, and we give a proof outline in Sections 4 and 5. Finally, we conclude in Section 6.

\section{Preliminaries}

\subsection{Basic notation}

Real vectors and matrices are represented by lower- and upper-case letters respectively. Boldface symbols denote random vectors, and their non-boldface counterparts denote particular realizations. $x^{\top}$ denotes the transpose of vector $x$. $\mathbb{E}$ denotes the expectation operator. We write $\mathbf{x}=\mathcal{N}(\mu, \Sigma)$ when $\mathbf{x}$ is a multivariate Gaussian random vector with mean $\mu$ and covariance $\Sigma$.

We consider discrete time stochastic processes over a finite time interval $[0, T]$. Time is indicated using subscripts, and we use the colon notation to denote ranges. For example: $x_{0: T-1}=\left\{x_{0}, x_{1}, \ldots, x_{T-1}\right\}$. In general, all symbols are time-varying. In an effort to present general results while keeping equations clear and concise, we introduce a new notation to represent a family of equations. For example, when we write:

$$
\mathbf{x}_{+} \stackrel{t}{=} A \mathbf{x}+\mathbf{w},
$$

we mean that $\mathbf{x}_{t+1}=A_{t} \mathbf{x}_{t}+\mathbf{w}_{t}$ holds for $0 \leq t \leq T-1$. Note that the subscript "+" indicates that we increment to $t+1$ for the associated symbol. We similarly overload the summation symbol by writing for example

$$
\sum_{t} x^{\top} Q x \quad \text { to mean } \sum_{t=0}^{T-1} x_{t}^{\top} Q_{t} x_{t}
$$

Whenever " $t$ " is written above a binary relation or below a summation, it is implied that $0 \leq t \leq T-1$. There is no ambiguity because we use the same time horizon $T$ throughout this paper.

We denote subvectors by using superscripts. Subvectors may also be referenced by using a subset of indices as superscripts. For example, for a vector

$$
\mathbf{x}=\left[\begin{array}{l}
\mathbf{x}^{1} \\
\mathbf{x}^{2} \\
\mathbf{x}^{3}
\end{array}\right], \quad \text { if } s=\{1,3\}, \text { we write: } \quad \mathbf{x}^{s}=\mathbf{x}^{\{1,3\}}=\left[\begin{array}{l}
\mathbf{x}^{1} \\
\mathbf{x}^{3}
\end{array}\right]
$$


When writing sub-vectors, we will always arrange the components in increasing order of indices. Given a collection of random vectors, we will at times treat the collection as a concatentation of vectors arranged in increasing order of node index.

For a matrix $A$, let $A_{i j}$ denotes its $(i, j)$ block with dimensions inferred from the context. Given two sets of indices $s$ and $r, A^{s, r}$ is a matrix composed of blocks $A_{i j}$ with $i \in s$ and $j \in r$.

\subsection{Graphs}

Let $G(\mathcal{V}, \mathcal{E})$ be a directed acyclic graph (DAG). The nodes are labeled 1 to $n$, so $\mathcal{V}=\{1, \ldots, n\}$. If there is an edge from $i$ to $j$, we write $(i, j) \in \mathcal{E}$.

We write $i \rightarrow j$ if there is a directed path from $i$ to $j$. That is, if there exists a sequence of nodes $v_{1}, \ldots, v_{m}$ with $v_{1}=i$ and $v_{m}=j$ such that $\left(v_{k}, v_{k+1}\right) \in \mathcal{E}$ for all $k$. By convention, every node has a directed path (of length zero) to itself. So it is always true that $i \rightarrow i$. We write $i \leftrightarrow j$ if $i$ and $j$ are path-connected, that is, if $i \rightarrow j$ or $j \rightarrow i$. Otherwise, we say they are path-disconnected, and we write $i \leftrightarrow j$. We can express the path-connectedness of $G$ using the sparsity matrix, which is the binary matrix $\mathbb{S} \in\{0,1\}^{n \times n}$ defined by

$$
\mathbb{S}_{i j}= \begin{cases}1 & \text { if } j \rightarrow i \\ 0 & \text { otherwise }\end{cases}
$$

Note that different graphs may have the same sparsity matrix. In general, $\mathbb{S}$ is the adjacency matrix of the transitive closure of $G$. So graphs with the same transitive closure also share the same sparsity matrix.

By convention, we assign a topological ordering to the node labels. That is, we choose a labeling such that if $j \rightarrow i$, then $j \leq i$. This is possible for any DAG $[3, \S 22.4]$. Therefore, $\mathbb{S}$ is always lower-triangular. For example, the sparsity matrix for the graph of Figure 1 is given by

$$
\mathbb{S}=\left[\begin{array}{lllll}
1 & 0 & 0 & 0 & 0 \\
0 & 1 & 0 & 0 & 0 \\
1 & 1 & 1 & 0 & 0 \\
0 & 1 & 0 & 1 & 0 \\
1 & 1 & 1 & 0 & 1
\end{array}\right]
$$

Given a node $i \in \mathcal{V}$, we define its ancestors as the set of nodes that have a directed path to $i$. Similarly, we define the descendants as the set of nodes that $i$ can reach via a directed path. We use the following notation for ancestors and descendants respectively.

$$
i^{\uparrow}=\{j \in \mathcal{V}: j \rightarrow i\} \quad i^{\downarrow}=\{j \in \mathcal{V}: i \rightarrow j\}
$$

Ancestors and descendants of $i$ always include $i$ itself. We define the strict ancestors and strict descendants when we mean to exclude $i$. Specifically, $i^{\uparrow}=i^{\uparrow} \backslash\{i\}$ and $i^{\downarrow}=i^{\downarrow} \backslash\{i\}$. We use the notation $i^{\uparrow}=i^{\uparrow} \cup i^{\downarrow}$ for the set of all nodes that are path-connected to node $i$. Note that $i^{\downarrow}$ is partitioned as $i^{\uparrow} \cup\{i\} \cup i^{\downarrow}$. In the graph of Figure 1, for example,

$$
\begin{gathered}
3^{\uparrow}=\{1,2,3\}, \quad 3^{\uparrow}=\{1,2\}, \quad 3^{\downarrow}=\{3,5\}, \\
3^{\downarrow}=\{5\}, \quad 3^{\natural}=\{1,2,3,5\}
\end{gathered}
$$

Remark 1. Note that while $i^{\uparrow}, i^{\downarrow}$, etc. are defined as subsets, it is convenient to think of them as ordered lists in which the node indices are arranged in increasing order. Thus, in Figure 1, $3^{\uparrow}$ will always be written as $\{1,2,3\}$ and not as any other permutation of $\{1,2,3\}$.

A node with no strict descendants is called a leaf node and a node with no strict ancestors is called a root node.

\subsection{System model}

The system we consider consists of $n$ subsystems that may affect one another according to the structure of an underlying DAG, $G(\mathcal{V}, \mathcal{E})$. The $i^{\text {th }}$ subsystem has state $\mathbf{x}^{i}$, input $\mathbf{u}^{i}$, measurement $\mathbf{y}^{i}$, process noise $\mathbf{w}^{i}$, and measurement noise $\mathbf{v}^{i}$. We assume these are discretetime random processes that satisfy the following statespace dynamics for all $i \in \mathcal{V}$.

$$
\begin{aligned}
& \mathbf{x}_{+}^{i} \stackrel{t}{=} \sum_{j \in i^{\uparrow}}\left(A_{i j} \mathbf{x}^{j}+B_{i j} \mathbf{u}^{j}\right)+\mathbf{w}^{i} \\
& \mathbf{y}^{i} \stackrel{t}{=} \sum_{j \in i^{\uparrow}}\left(C_{i j} \mathbf{x}^{j}\right)+\mathbf{v}^{i}
\end{aligned}
$$

The relative timing of $i^{\text {th }}$ state, control action and observation at time $t$ is as shown in Figure 2. Note that the observation $\mathbf{y}_{t}^{i}$ is generated after control action $\mathbf{u}_{t}^{i}$ is taken. Each of the matrices in (1) may be time-varying,

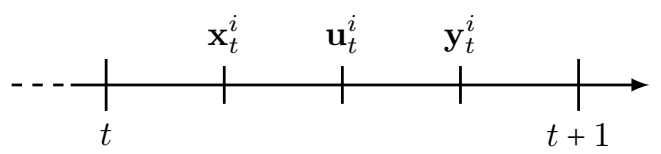

Figure 2: Relative timing of state, action and observation at time $t$.

and may even change dimensions with time. In an effort to make our notation more concise, we concatenate the various symbols above and simply write

$$
\begin{gathered}
\mathbf{x}_{+} \stackrel{t}{=} A \mathbf{x}+B \mathbf{u}+\mathbf{w} \\
\mathbf{y} \stackrel{t}{=} C \mathbf{x}+\mathbf{v}
\end{gathered}
$$

In this condensed notation, the matrices $A, B$, and $C$ have blocks that conform to $\mathbb{S}$, the sparsity matrix for $G(\mathcal{V}, \mathcal{E})$. In other words, if $\mathbb{S}_{i j}=0$ then $A_{i j}=0, B_{i j}=0$, and $C_{i j}=0$. The random vectors in the collection

$$
\left\{\mathbf{x}_{0},\left[\begin{array}{l}
\mathbf{w}_{0} \\
\mathbf{v}_{0}
\end{array}\right], \ldots,\left[\begin{array}{l}
\mathbf{w}_{T-1} \\
\mathbf{v}_{T-1}
\end{array}\right]\right\}
$$


are referred to as the primitive random variables and are mutually independent and jointly Gaussian with the following known probability density functions

$$
\begin{gathered}
\mathbf{x}_{0}=\mathcal{N}\left(0, \Sigma_{\text {init }}\right) \\
{\left[\begin{array}{l}
\mathbf{w} \\
\mathbf{v}
\end{array}\right] \stackrel{t}{=} \mathcal{N}\left(0,\left[\begin{array}{cc}
W & U^{\top} \\
U & V
\end{array}\right]\right)}
\end{gathered}
$$

There are $n$ controllers, one responsible for each of the $\mathbf{u}^{i}$, $i=1, \ldots, n$. We define the locally generated information at node $i$ at time $t$ as

$$
\mathbf{i}_{t}^{i}:=\left\{\mathbf{y}_{0: t-1}^{i}, \mathbf{u}_{0: t-1}^{i}\right\}
$$

The information available to controller $i$ at time $t$ is

$$
\mathbf{i}_{t}^{i^{\uparrow}}:=\bigcup_{j \in i^{\uparrow}} \mathbf{i}_{t}^{j}=\bigcup_{j \in i^{\uparrow}}\left\{\mathbf{y}_{0: t-1}^{j}, \mathbf{u}_{0: t-1}^{j}\right\} .
$$

In other words, each controller knows the past measurements and decisions of its ancestors. So the directed edges of $G$ may be thought of as representing the flow of information between subsystems. Crucially, the same graph $G$ represents both how the dynamics propagate as well as how information is shared in the system. The controllers select actions according to a control strategy $f^{i}:=\left(f_{0}^{i}, f_{1}^{i}, \ldots, f_{T-1}^{i}\right)$ for $i \in \mathcal{V}$. That is,

$$
\mathbf{u}_{t}^{i}=f_{t}^{i}\left(\mathbf{i}_{t}^{i^{\uparrow}}\right) \quad \text { for } 0 \leq t \leq T-1
$$

Given a control strategy profile $f=\left(f^{1}, f^{2}, \ldots, f^{n}\right)$, performance is measured by the finite horizon expected quadratic cost

$$
\hat{\mathcal{J}}_{0}(f)=\mathbb{E}^{f}\left(\sum_{t}\left[\begin{array}{l}
\mathbf{x} \\
\mathbf{u}
\end{array}\right]^{\top}\left[\begin{array}{cc}
Q & S \\
S^{\top} & R
\end{array}\right]\left[\begin{array}{l}
\mathbf{x} \\
\mathbf{u}
\end{array}\right]+\mathbf{x}_{T}^{\top} P_{\text {final }} \mathbf{x}_{T}\right)
$$

Expectation is taken with respect to the joint probability measure on $\left(\mathbf{x}_{0: T}, \mathbf{u}_{0: T-1}\right)$ induced by the choice of $f$.

It is assumed that all system parameters are universally known. Specifically, $\Sigma_{\text {init }}, P_{\text {final }}$, as well as the values of $A, B, C, Q, R, S, U, V, W$ for all $t$, are known by all controllers.

\subsection{Assumptions}

In addition to the problem specifications (2)-(8), we will make some additional assumptions about the underlying DAG and the noise and cost parameters used in (4) and (8) respectively. First, we require some definitions.

Definition 1 (multitree). The nodes $i, a, b, j \in \mathcal{V}$ form $a$ diamond if $i \rightarrow a \rightarrow j$ and $i \rightarrow b \rightarrow j$, and $a \leftrightarrow b$. A multitree is a directed acyclic graph that contains no diamonds.

For example, the graph of Figure 1 is a multitree. However, if we add the edge $(4,5)$, then the nodes $(2,3,4,5)$ form a diamond and the graph ceases to be a multitree.
Definition 2 (decoupled cost). Define the set $\mathcal{X}=$ $\left\{Q_{0: T-1}, R_{0: T-1}, S_{0: T-1}, P_{\text {final }}\right\}$ of matrices associated with the cost function. We say that nodes $i, j \in \mathcal{V}$ have decoupled cost if $X_{i j}=0$ for all $X \in \mathcal{X}$.

Definition 3 (uncorrelated noise). Define the set $\mathcal{Y}=$ $\left\{W_{0: T-1}, V_{0: T-1}, U_{0: T-1}, \Sigma_{\text {init }}\right\}$ of matrices associated with the noise and initial state statistics. We say that nodes $i, j \in \mathcal{V}$ have uncorrelated noise if $Y_{i j}=0$ for all $Y \in \mathcal{Y}$.

The notions of decoupled cost and uncorrelated noise have intuitive interpretations. If two nodes have decoupled cost, then the instantaneous cost at any time has no cross-terms that involve both nodes. If two nodes have uncorrelated noise, then the process and measurement noises affecting one node are statistically independent of those affecting the other. Our assumptions are as follows.

(A1) The DAG $G(\mathcal{V}, \mathcal{E})$ is a multitree.

(A2) For every pair of nodes $i, j \in \mathcal{V}$,

- If the pair of nodes has no common ancestor, then they have uncorrelated noise.

- If the pair of nodes has no common descendants, then they have decoupled costs.

For the graph of Figure 1, nodes 1 and 4 have neither a common ancestor nor a common descendant. Therefore, Assumption A2 would require that this pair of nodes have both decoupled cost and uncorrelated noise.

Note that because of the multitree assumption, the only way $i$ and $j$ can have both a common ancestor and a common descendant is if $i \leftrightarrow j$. Assumption A2 may be expressed in terms of the sparsity pattern $\mathbb{S}$ using the following observations

1. $\left(\mathbb{S S}^{\top}\right)_{i j}=0$ if and only if $i^{\uparrow} \cap j^{\uparrow}=\varnothing$; in other words, if and only if $i$ and $j$ have no common ancestor.

2. $\left(\mathbb{S}^{\top} \mathbb{S}\right)_{i j}=0$ if and only if $i^{\downarrow} \cap j^{\downarrow}=\varnothing$; in other words, if and only if $i$ and $j$ have no common descendant.

So Assumption A2 may be stated concisely as follows: all matrices in $\mathcal{X}$ (see Definition 2) have the same sparsity as $\mathbb{S}^{\top} \mathbb{S}$ and all matrices in $\mathcal{Y}$ (see Definition 3 ) have the same sparsity as $\mathbb{S S}^{\top}$. For the graph of Figure 1, these sparsity patterns are

$$
\mathbb{S S}^{\top} \sim\left[\begin{array}{lllll}
1 & 0 & 1 & 0 & 1 \\
0 & 1 & 1 & 1 & 1 \\
1 & 1 & 1 & 1 & 1 \\
0 & 1 & 1 & 1 & 1 \\
1 & 1 & 1 & 1 & 1
\end{array}\right] \quad \mathbb{S}^{\top} \mathbb{S} \sim\left[\begin{array}{ccccc}
1 & 1 & 1 & 0 & 1 \\
1 & 1 & 1 & 1 & 1 \\
1 & 1 & 1 & 0 & 1 \\
0 & 1 & 0 & 1 & 0 \\
1 & 1 & 1 & 0 & 1
\end{array}\right]
$$

Remark 2. Note that Assumption A2 is more general than assuming that all cost matrices in $\mathcal{X}$ and covariance matrices in $\mathcal{Y}$ are block-diagonal. 


\section{Main results}

The problem addressed in this paper is the following.

Problem 1 ( $n$-player LQG). For the model (2)-(7), and subject to Assumptions $A 1$ and A2, find a control strategy profile $f=\left(f^{1}, f^{2}, \ldots, f^{n}\right)$ that minimizes the expected cost (8).

The information structure of our problem is partially nested and therefore, without loss of optimality, we will restrict attention to linear control strategies [4]. The main result of this paper is a description of sufficient statistics required for an optimal solution of Problem 1.

Theorem 1 (Control Result). In Problem 1, there is no loss in optimality in jointly restricting all nodes $i \in \mathcal{V}$ to strategies of the form

$$
\mathbf{u}_{t}^{i}=\sum_{j \in i^{\uparrow}} K_{t}^{i j} \mathbf{z}_{t}^{j^{\downarrow}}
$$

where $\mathbf{z}_{t}^{j^{\downarrow}}:=\mathbb{E}\left(\mathbf{x}_{t}^{j^{\downarrow}} \mid \mathbf{i}_{t}^{j^{\uparrow}}\right)$.

Note that $\mathbf{z}_{t}^{j^{\downarrow}}$ is the conditional mean of the state of all nodes that are path-connected to node $j$ based on the information available to node $j$ (recall the notation introduced in Section 2.2).

Recall that $\mathbf{i}_{t}^{i^{\uparrow}}$ defined in (5)-(6) is the information available to node $i$ at time $t$, and this set grows with time as more measurements are observed and more decisions are made. Theorem 1 states that controllers need not remember this entire information history. Instead, each node $j$ may compute the aggregated statistic $\mathbf{z}^{j^{\downarrow}}$, which is an estimate of the current states of its ancestors and descendants. The optimal decision at node $i, \mathbf{u}^{i}$, is then a linear function of all such estimates maintained by the ancestors of node $i$.

Our second result addresses the evolution of the estimates $\mathbf{z}_{t}^{j^{\downarrow}}$. Before we state this result, we define the set of matrices $E^{i, j}$.

Definition 4. Consider nodes $i$ and $j$ with $i \in j^{\Downarrow}$. Let $i^{\downarrow}=\left\{k_{1}, k_{2}, \ldots, k_{|i \downarrow|}\right\}$ and $j^{\downarrow}=\left\{l_{1}, l_{2}, \ldots, l_{\mid j \downarrow}\right\}$. Define $a$ matrix $E^{i, j}$ with $\left|i^{\uparrow}\right|$ block rows and $\left|j^{\uparrow}\right|$ block columns as follows: For $a=1,2, \ldots,\left|i^{\uparrow}\right|$,

1. If $k_{a} \notin j^{\uparrow}$, then the $a^{\text {th }}$ block row of $E^{i, j}$ is 0 .

2. If $k_{a} \in j^{\uparrow}$ and $k_{a}=l_{b}$, then the $(a, b)$ block of $E^{i, j}$ is identity and the rest of $a^{\text {th }}$ block row is 0 .

For example, in Figure 1, we have $3^{\downarrow}=\{1,2,3,5\}$ and $2^{\uparrow}=\{2,3,4,5\}$. Consequently,

$$
E^{3,2}=\left[\begin{array}{cccc}
0 & 0 & 0 & 0 \\
I & 0 & 0 & 0 \\
0 & I & 0 & 0 \\
0 & 0 & 0 & I
\end{array}\right]
$$

We now state our second result.

Theorem 2 (Estimation Result). If the control strategy is as given in Theorem 1 , then the evolution of $\mathbf{z}_{t}^{j^{\downarrow}}$ is described as follows:

$$
\begin{aligned}
& \mathbf{z}_{0}^{j^{\downarrow}}=0 \\
& \mathbf{z}_{+}^{j^{\downarrow}} \stackrel{t}{=} A^{j^{\downarrow} j^{\downarrow}} \mathbf{z}^{j^{\downarrow}}+B^{j^{\downarrow} j^{\downarrow}}\left[\begin{array}{c}
\mathbf{u}^{j^{\uparrow}} \\
\left\{\hat{\mathbf{u}}^{i j}\right\}_{i \in j \Downarrow}
\end{array}\right]-L^{j}\left(\mathbf{y}^{j^{\uparrow}}-C^{j^{\uparrow} j^{\uparrow}} \mathbf{z}^{j^{\uparrow}}\right)
\end{aligned}
$$

for some matrices $L_{0: T-1}^{j}$, with

$$
\hat{\mathbf{u}}^{i j}: \stackrel{t}{=} \sum_{a \in j^{\uparrow}} K^{i a} \mathbf{z}^{a^{\downarrow}}+\sum_{b \in i^{\uparrow} \cap j \downarrow} K^{i b} E^{b, j} \mathbf{z}^{j^{\downarrow}} \quad \text { for } \quad i \in j^{\downarrow}
$$

Remark 3. For linear control strategies described by Theorem 2, the quantity $\hat{\mathbf{u}}_{t}^{i j}$ as defined in (11) is in fact equal to $\mathbb{E}\left(\mathbf{u}_{t}^{i} \mid \mathbf{i}_{t}^{j^{\uparrow}}\right)$.

The above theorems provide finite dimensional sufficient statistics for all controllers in the system. These results should be viewed as structural results of optimal control strategies since they postulate the existence of optimal controllers and estimators of the form presented above without specifying how the matrices $K^{i j}, L^{j}$ used in control and estimation can be computed.

\section{Proof outline of Theorem 1 for the system in Figure 1}

Our proof technique may be thought of as a sequence of refinements that traverses the underlying DAG starting from the leaf nodes and finishing at the root nodes.

Due to space limitations, we will present a proof outline of Theorem 1 as applied to the system shown in Figure 1. The most basic structural form of control strategies for the system illustrated in Figure 1 is simply our initial information constraint (7), namely, $\mathbf{u}_{t}^{i}=f_{t}^{i}\left(\mathbf{i}_{t}^{i^{\uparrow}}\right)$. We refine this structural form in the following steps:

1. Leaf nodes 4 and 5: We consider a single leaf node (say node 4) and fix arbitrary linear control strategies for all nodes except node 4 . We will consider the problem of finding the best control strategy for node 4 in response to the arbitrary choice of linear control strategies of all other controllers. This is a centralized control problem for which we can derive a structural result by identifying a suitable state description for the overall system as seen by node 4 . With fixed linear strategies for all other nodes, the overall system can be viewed as a LQG system with $\mathbf{x}_{t}^{1,2,3,4,5}, \mathbf{i}_{t}^{1,2,3,5}$ as the state vector and $\mathbf{y}_{t}^{2,4}, \mathbf{u}_{t}^{2}$ as the observation vector. Because of the structure of the graph and the sparsity assumptions on the cost matrices, the dynamics and the cost terms associated with nodes $1,3,5$ are decoupled from the dynamics and costs associated with node 4 . Therefore, for node 4's centralized problem, it suffices to consider $\mathbf{x}_{t}^{2,4}, \mathbf{i}_{t}^{2}$ as the state. From 
standard LQG theory [6], it follows that node 4 can use a linear control law that is a function of its estimate of state vector $\mathbf{x}_{t}^{2,4}, \mathbf{i}_{t}^{2}$. That is, node 4's linear control law is of the form

$$
\mathbf{u}_{t}^{4}=g_{t}^{4}\left(\mathbf{z}_{t}^{4^{\uparrow}}\right)+h_{t}^{42}\left(\mathbf{i}_{t}^{2}\right)
$$

where $\mathbf{z}_{t}^{4^{\uparrow}}:=\mathbb{E}\left(\mathbf{x}_{t}^{2,4} \mid \mathbf{i}_{t}^{2,4}\right)$. A similar argument for node 5 yields the structure

$$
\begin{aligned}
\mathbf{u}_{t}^{5} & =g_{t}^{5}\left(\mathbf{z}_{t}^{5^{\uparrow}}\right)+h_{t}^{5}\left(\mathbf{i}_{t}^{1,2,3}\right) \\
& =g_{t}^{5}\left(\mathbf{z}_{t}^{5^{\uparrow}}\right)+h_{t}^{51}\left(\mathbf{i}_{t}^{1}\right)+h_{t}^{52}\left(\mathbf{i}_{t}^{2}\right)+h_{t}^{53}\left(\mathbf{i}_{t}^{3}\right)
\end{aligned}
$$

where $\mathbf{z}_{t}^{5^{\uparrow}}:=\mathbb{E}\left(\mathbf{x}_{t}^{1,2,3,5} \mid \mathbf{i}_{t}^{1,2,3,5}\right)$.

2. Parent of leaf nodes; node 3 : Next we consider nodes with only leaf nodes as children. In Figure 1, the only such node is node 3 . We fix arbitrary linear strategies for nodes 1 and 2 . For node 4, we pick any strategy that has the structural form (12) identified in Step 1. We turn our attention to optimizing strategies for node 3 and its descendant, node 5. From Step 1, we know that

$$
\begin{aligned}
& \mathbf{u}_{t}^{3}=f_{t}^{3}\left(\mathbf{i}_{t}^{1,2,3}\right) \\
& \mathbf{u}_{t}^{5}=g_{t}^{5}\left(\mathbf{z}_{t}^{5^{\uparrow}}\right)+h_{t}^{5}\left(\mathbf{i}_{t}^{1,2,3}\right)
\end{aligned}
$$

We consider arbitrary choices for functions $g_{0: T-1}^{5}$ in (14) and focus on the joint optimization of $f_{0: T-1}^{3}$ and $h_{0: T-1}^{5}$. The key thing to note here is that both $f_{t}^{3}$ and $h_{t}^{5}$ are functions of information available to node 3 . Therefore, we will introduce a fictitious coordinator for nodes 3 and 5 that knows $\mathbf{i}_{t}^{1,2,3}$ and selects two decisions:

$$
\begin{aligned}
\mathbf{u}_{t}^{3} & =f_{t}^{3}\left(\mathbf{i}_{t}^{1,2,3}\right) \\
\tilde{\mathbf{u}}_{t}^{53} & =h_{t}^{5}\left(\mathbf{i}_{t}^{1,2,3}\right)
\end{aligned}
$$

so that the control action at node 5 can be written as

$$
\mathbf{u}_{t}^{5}=g_{t}^{5}\left(\mathbf{z}_{t}^{5^{\uparrow}}\right)+\tilde{\mathbf{u}}_{t}^{53}
$$

We now consider the centralized problem of how this coordinator should optimally make its decisions.

With strategies (or parts of strategies) of other controllers fixed, the overall system can be viewed as a LQG system from the coordinator's perspective with $\mathbf{x}_{t}^{1,2,3,4,5}, \mathbf{i}_{t}^{1,2}, \mathbf{z}_{t}^{4^{\uparrow}}, \mathbf{z}_{t}^{5^{\uparrow}}$ as the state vector and $\mathbf{y}_{t}^{1,3}, \mathbf{u}_{t}^{1}$ as the observation vector. Because of the structure of the graph and the sparsity assumptions on the cost matrices, the dynamics and the cost terms associated with nodes $1,3,5$ are decoupled from the dynamics and costs associated with node 4 . Therefore, for the coordinator's centralized problem, it suffices to consider $\mathbf{x}_{t}^{1,2,3,5}, \mathbf{i}_{t}^{1,2}, \mathbf{z}_{t}^{5^{\uparrow}}$ as the state. Using the same argument as in Step 1, it suffices that the coordinator estimate this new state. The estimate of $\mathbf{z}_{t}^{5^{\uparrow}}$ can be written as

$$
\mathbb{E}\left(\mathbf{z}_{t}^{5^{\uparrow}} \mid \mathbf{i}_{t}^{1,2,3}\right)=\mathbb{E}\left(\mathbb{E}\left(\mathbf{x}_{t}^{1,2,3,5} \mid \mathbf{i}_{t}^{1,2,3,5}\right) \mid \mathbf{i}_{t}^{1,2,3}\right)
$$

By the smoothing property of conditional expectation,

$$
\mathbb{E}\left(\mathbf{z}_{t}^{5^{\uparrow}} \mid \mathbf{i}_{t}^{1,2,3}\right)=\mathbb{E}\left(\mathbf{x}_{t}^{1,2,3,5} \mid \mathbf{i}_{t}^{1,2,3}\right)
$$

Therefore, the fictitious coordinator's optimal strategy can be written as

$$
\begin{gathered}
\mathbf{u}_{t}^{3}=g_{t}^{3}\left(\mathbf{z}_{t}^{3^{\downarrow}}\right)+h_{t}^{31}\left(\mathbf{i}_{t}^{1}\right)+h_{t}^{32}\left(\mathbf{i}_{t}^{2}\right) \\
\tilde{\mathbf{u}}_{t}^{53}=g_{t}^{53}\left(\mathbf{z}_{t}^{3^{\downarrow}}\right)+h_{t}^{51}\left(\mathbf{i}_{t}^{1}\right)+h_{t}^{52}\left(\mathbf{i}_{t}^{2}\right)
\end{gathered}
$$

where $\mathbf{z}_{t}^{3^{\downarrow}}=\mathbb{E}\left(\mathbf{x}_{t}^{1,2,3,5} \mid \mathbf{i}_{t}^{1,2,3}\right)$. Combining (15) with (18) yields the following structure for $\mathbf{u}_{t}^{3}$ and $\mathbf{u}_{t}^{5}$

$$
\begin{aligned}
& \mathbf{u}_{t}^{3}=g_{t}^{3}\left(\mathbf{z}_{t}^{3^{\uparrow}}\right)+h_{t}^{31}\left(\mathbf{i}_{t}^{1}\right)+h_{t}^{32}\left(\mathbf{i}_{t}^{2}\right) \\
& \mathbf{u}_{t}^{5}=g_{t}^{5}\left(\mathbf{z}_{t}^{5^{\uparrow}}\right)+g_{t}^{53}\left(\mathbf{z}_{t}^{3^{\uparrow}}\right)+h_{t}^{51}\left(\mathbf{i}_{t}^{1}\right)+h_{t}^{52}\left(\mathbf{i}_{t}^{2}\right)
\end{aligned}
$$

3. Root nodes 1 and 2: Finally, we consider the roots nodes 1 and 2 . We start with node 2 . We fix arbitrary linear strategies for node 1 and focus on optimizing strategies for node 2 and its descendants. Collecting the results from Steps 1 and 2, we have

$$
\begin{aligned}
& \mathbf{u}_{t}^{2}=f_{t}^{2}\left(\mathbf{i}_{t}^{2}\right) \\
& \mathbf{u}_{t}^{3}=g_{t}^{3}\left(\mathbf{z}_{t}^{3^{\uparrow}}\right)+h_{t}^{31}\left(\mathbf{i}_{t}^{1}\right)+h_{t}^{32}\left(\mathbf{i}_{t}^{2}\right) \\
& \mathbf{u}_{t}^{4}=g_{t}^{4}\left(\mathbf{z}_{t}^{4^{\uparrow}}\right)+h_{t}^{42}\left(\mathbf{i}_{t}^{2}\right) \\
& \mathbf{u}_{t}^{5}=g_{t}^{5}\left(\mathbf{z}_{t}^{5^{\uparrow}}\right)+g_{t}^{53}\left(\mathbf{z}_{t}^{3^{\uparrow}}\right)+h_{t}^{51}\left(\mathbf{i}_{t}^{1}\right)+h_{t}^{52}\left(\mathbf{i}_{t}^{2}\right)
\end{aligned}
$$

Fix arbitrary choices for $g_{t}^{3}, g_{t}^{4}, g_{t}^{5}, h_{t}^{31}, h_{t}^{51}, t=0, \ldots, T-1$ and focus on the joint optimization of $f_{t}^{2}, h_{t}^{32}, h_{t}^{42}, h_{t}^{52}$ for each $t=0, \ldots, T-1$. Note that all the functions being optimized here are functions of $\mathbf{i}_{t}^{2}$, the information available to node 2. As in Step 2, introduce a coordinator for nodes $2,3,4,5$ that knows $\mathbf{i}_{t}^{2}$ and selects

$$
\begin{array}{cl}
\mathbf{u}_{t}^{2}=f_{t}^{2}\left(\mathbf{i}_{t}^{2}\right) & \tilde{\mathbf{u}}_{t}^{42}=h_{t}^{42}\left(\mathbf{i}_{t}^{2}\right) \\
\tilde{\mathbf{u}}_{t}^{32}=h_{t}^{32}\left(\mathbf{i}_{t}^{2}\right) & \tilde{\mathbf{u}}_{t}^{52}=h_{t}^{52}\left(\mathbf{i}_{t}^{2}\right)
\end{array}
$$

With strategies (or parts of strategies) of other controllers fixed, the overall system can be viewed as a LQG system from the new coordinator's perspective with $\mathbf{x}_{t}^{1,2,3,4,5}, \mathbf{i}_{t}^{1}, \mathbf{z}_{t}^{3^{\uparrow}}, \mathbf{z}_{t}^{4^{\uparrow}}, \mathbf{z}_{t}^{5^{\uparrow}}$ as the state vector and $\mathbf{y}_{t}^{2}$ as the observation vector. It suffices for the coordinator to estimate this state. We now make the following observation:

a) Because of the sparsity assumption about noise statistics (see Assumption A2), $\mathbf{x}_{t}^{1}, \mathbf{i}_{t}^{1}$ are independent of the coordinator's information $\mathbf{i}_{t}^{2}$ at time $t$. Therefore, $\mathbb{E}\left(\mathbf{x}_{t}^{1} \mid \mathbf{i}_{t}^{2}\right)=\mathbf{0}$ and $\mathbb{E}\left(\mathbf{i}_{t}^{1} \mid \mathbf{i}_{t}^{2}\right)=\mathbf{0}$.

b) The coordinator's estimate of $\mathbf{z}_{t}^{4^{\uparrow}}$ can be written as

$$
\mathbb{E}\left(\mathbf{z}_{t}^{4^{\uparrow}} \mid \mathbf{i}_{t}^{2}\right)=\mathbb{E}\left(\mathbb{E}\left(\mathbf{x}_{t}^{2,4} \mid \mathbf{i}_{t}^{2,4}\right) \mid \mathbf{i}_{t}^{2}\right)=\mathbb{E}\left(\mathbf{x}_{t}^{2,4} \mid \mathbf{i}_{t}^{2}\right)
$$

c) The coordinator's estimate of $\mathbf{z}_{t}^{3^{\downarrow}}$ can be written as

$$
\mathbb{E}\left(\mathbf{z}_{t}^{3^{\mathfrak{}}} \mid \mathbf{i}_{t}^{2}\right)=\mathbb{E}\left(\mathbb{E}\left(\mathbf{x}_{t}^{1,2,3,5} \mid \mathbf{i}_{t}^{1,2,3}\right) \mid \mathbf{i}_{t}^{2}\right)=\mathbb{E}\left(\mathbf{x}_{t}^{1,2,3,5} \mid \mathbf{i}_{t}^{2}\right)
$$


Furthermore,

$$
\begin{aligned}
& \mathbb{E}\left(\mathbf{x}_{t}^{1,2,3,5} \mid \mathbf{i}_{t}^{2}\right) \\
& =\mathbb{E}\left(\mathbf{x}_{t}^{1,2,3,5}-E^{3,2} \mathbf{x}_{t}^{2,3,4,5} \mid \mathbf{i}_{t}^{2}\right)+\mathbb{E}\left(E^{3,2} \mathbf{x}_{t}^{2,3,4,5} \mid \mathbf{i}_{t}^{2}\right) \\
& =\mathbb{E}\left(\left[\begin{array}{llll}
\mathbf{x}_{t}^{1} & \mathbf{0} & \mathbf{0} & \mathbf{0}
\end{array}\right]^{\top} \mid \mathbf{i}_{t}^{2}\right)+E^{3,2} \mathbb{E}\left(\mathbf{x}_{t}^{2,3,4,5} \mid \mathbf{i}_{t}^{2}\right) \\
& =\mathbf{0}+E^{3,2} \mathbb{E}\left(\mathbf{x}_{t}^{2,3,4,5} \mid \mathbf{i}_{t}^{2}\right)
\end{aligned}
$$

d) Similarly,

$$
\mathbb{E}\left(\mathbf{z}_{t}^{5^{\uparrow}} \mid \mathbf{i}_{t}^{2}\right)=E^{5,2} \mathbb{E}\left(\mathbf{x}_{t}^{2,3,4,5} \mid \mathbf{i}_{t}^{2}\right)
$$

Based on (21)-(24), we observe that the estimate of the new coordinator's state ultimately only depends on $\mathbb{E}\left(\mathbf{x}_{t}^{2,3,4,5} \mid \mathbf{i}_{t}^{2}\right)$. Therefore, (20) can be refined to

$$
\begin{aligned}
\mathbf{u}_{t}^{2}=f_{t}^{2}\left(\mathbf{z}_{t}^{2^{\natural}}\right) & \tilde{\mathbf{u}}_{t}^{42}=g_{t}^{42}\left(\mathbf{z}_{t}^{2^{\natural}}\right) \\
\tilde{\mathbf{u}}_{t}^{32}=g_{t}^{32}\left(\mathbf{z}_{t}^{2^{\natural}}\right) & \tilde{\mathbf{u}}_{t}^{52}=g_{t}^{52}\left(\mathbf{z}_{t}^{2^{\natural}}\right)
\end{aligned}
$$

where $\mathbf{z}_{t}^{2^{\natural}}=\mathbb{E}\left(\mathbf{x}_{t}^{2,3,4,5} \mid \mathbf{i}_{t}^{2}\right)$. Now merge (25) with (19) and obtain the following structure for $\mathbf{u}_{t}^{2}, \mathbf{u}_{t}^{3}, \mathbf{u}_{t}^{4}, \mathbf{u}_{t}^{5}$

$$
\begin{aligned}
& \mathbf{u}_{t}^{2}=g_{t}^{2}\left(\mathbf{z}_{t}^{2^{\uparrow}}\right) \\
& \mathbf{u}_{t}^{3}=g_{t}^{3}\left(\mathbf{z}_{t}^{3^{\downarrow}}\right)+g_{t}^{32}\left(\mathbf{z}_{t}^{2^{\downarrow}}\right)+h_{t}^{31}\left(\mathbf{i}_{t}^{1}\right) \\
& \mathbf{u}_{t}^{4}=g_{t}^{4}\left(\mathbf{z}_{t}^{4^{\uparrow}}\right)+g_{t}^{42}\left(\mathbf{z}_{t}^{2^{\uparrow}}\right) \\
& \mathbf{u}_{t}^{5}=g_{t}^{5}\left(\mathbf{z}_{t}^{5^{\uparrow}}\right)+g_{t}^{52}\left(\mathbf{z}_{t}^{2^{\uparrow}}\right)+g_{t}^{53}\left(\mathbf{z}_{t}^{3^{\uparrow}}\right)+h_{t}^{51}\left(\mathbf{i}_{t}^{1}\right)
\end{aligned}
$$

Repeating a similar argument for node 1 establishes the final structural result for all nodes.

$$
\begin{aligned}
& \mathbf{u}_{t}^{1}=g_{t}^{1}\left(\mathbf{z}_{t}^{1^{\uparrow}}\right) \\
& \mathbf{u}_{t}^{2}=g_{t}^{2}\left(\mathbf{z}_{t}^{2^{\natural}}\right) \\
& \mathbf{u}_{t}^{4}=g_{t}^{4}\left(\mathbf{z}_{t}^{4^{\uparrow}}\right)+g_{t}^{42}\left(\mathbf{z}_{t}^{2^{\uparrow}}\right) \\
& \mathbf{u}_{t}^{3}=g_{t}^{3}\left(\mathbf{z}_{t}^{3^{\mathfrak{1}}}\right)+g_{t}^{31}\left(\mathbf{z}_{t}^{1^{\mathfrak{1}}}\right)+g_{t}^{32}\left(\mathbf{z}_{t}^{2^{\mathfrak{1}}}\right) \\
& \mathbf{u}_{t}^{5}=g_{t}^{5}\left(\mathbf{z}_{t}^{5^{\dagger}}\right)+g_{t}^{51}\left(\mathbf{z}_{t}^{\mathbf{1}^{\downarrow}}\right)+g_{t}^{52}\left(\mathbf{z}_{t}^{2^{\downarrow}}\right)+g_{t}^{53}\left(\mathbf{z}_{t}^{3^{\downarrow}}\right)
\end{aligned}
$$

Observing that all functions in (27) are linear and therefore can be written in terms of matrices yields the result of Theorem 1 for the system of Figure 1.

\section{$5 \quad$ Proof outline of Theorem 2}

The steps 1-3 in the proof of Theorem 1 invoke the centralized structural result from standard LQG theory [6]; a sufficient statistic for the optimal controller is the conditional mean of the state. But the centralized theory also provides a recursive formulation for the conditional mean (the Kalman filter). Theorem 2 can be proved by augmenting the proof of Theorem 1 to include these estimator recursions at every step. We provide an outline below and refer the reader to [16] for a detailed proof.
Consider first a leaf node, say node 4 . This node needs to evaluate $\mathbf{z}_{t}^{4^{\dagger}}:=\mathbb{E}\left(\mathbf{x}_{t}^{2,4} \mid \mathbf{i}_{t}^{2,4}\right)$. The structure of the graph implies that the states of nodes 2 and $4, \mathbf{x}_{t}^{2,4}$, evolve according to the following dynamics:

$$
\begin{aligned}
& \mathbf{x}_{+}^{2} \stackrel{t}{=} A_{22} \mathbf{x}^{2}+B_{22} \mathbf{u}^{2}+\mathbf{w}^{2} \\
& \mathbf{x}_{+}^{4} \stackrel{t}{=} A_{42} \mathbf{x}^{2}+A_{44} \mathbf{x}^{4}+B_{42} \mathbf{u}^{2}+B_{44} \mathbf{u}^{4}+\mathbf{w}^{4} \\
& \mathbf{y}^{2} \stackrel{t}{=} C_{22} \mathbf{x}^{2}+\mathbf{v}^{2} \\
& \mathbf{y}^{4} \stackrel{t}{=} C_{42} \mathbf{x}^{2}+C_{44} \mathbf{x}^{4}+\mathbf{v}^{i}
\end{aligned}
$$

Computing the estimator of these states based on $\mathbf{i}_{t}^{2,4}=$ $\left\{\mathbf{y}_{0: t-1}^{2,4}, \mathbf{u}_{0: t-1}^{2,4}\right\}$ is a standard Kalman estimation problem with a recursive update equation that agrees with the result of Theorem 2 for $j=4$. The estimates at the other leaf node, node 5 , can be handled in a similar manner.

Now focus on node 3 's estimate $\mathbf{z}_{t}^{3^{\downarrow}}:=\mathbb{E}\left(\mathbf{x}_{t}^{1,2,3,5} \mid \mathbf{i}_{t}^{1,2,3}\right)$. The difficulty in updating this estimate is that the dynamics of $\mathbf{x}_{t}^{5}$ depend on node 5's control action which node 3 does not fully know. Because of Theorem 1, the action of node 5 can be written as

$$
\mathbf{u}_{t}^{5}=K_{t}^{55} \mathbf{z}_{t}^{5^{\uparrow}}+K_{t}^{53} \mathbf{z}_{t}^{3^{\uparrow}}+K_{t}^{52} \mathbf{z}_{t}^{2^{\uparrow}}+K_{t}^{51} \mathbf{z}_{t}^{5^{\uparrow}}
$$

The last three terms in (29) are based on information available to node 3 and are therefore computable by node 3 . To estimate $\mathbf{u}_{t}^{5}$, therefore, node 3 needs to estimate the first term. As we saw in proof of Theorem 1,

$$
\mathbb{E}\left(\mathbf{z}_{t}^{5^{\uparrow}} \mid \mathbf{i}_{t}^{1,2,3}\right)=\mathbb{E}\left(\mathbf{x}_{t}^{1,2,3,5} \mid \mathbf{i}_{t}^{1,2,3}\right)=\mathbf{z}_{t}^{3^{\uparrow}}
$$

Using the above observations, we can derive the following update equation for $\mathbf{z}_{t}^{3^{\downarrow}}$ :

$$
\mathbf{z}_{+}^{3^{\uparrow}} \stackrel{t}{=} A^{3^{\uparrow} 3^{\uparrow}} \mathbf{z}^{3^{\downarrow}}+B^{3^{\uparrow} 3^{\downarrow}}\left[\begin{array}{c}
\mathbf{u}^{3^{\uparrow}} \\
\hat{\mathbf{u}}^{53}
\end{array}\right]-L^{3}\left(\mathbf{y}^{3^{\uparrow}}-C^{3^{\uparrow} 3^{\uparrow}} \mathbf{z}^{3^{\uparrow}}\right)
$$

for some matrices $L_{0: T-1}^{3}$, with

$$
\hat{\mathbf{u}}^{53} \stackrel{t}{=} K_{t}^{55} \mathbf{z}_{t}^{3^{\uparrow}}+K_{t}^{53} \mathbf{z}_{t}^{3^{\downarrow}}+K_{t}^{52} \mathbf{z}_{t}^{2^{\downarrow}}+K_{t}^{51} \mathbf{z}_{t}^{5^{\downarrow}}
$$

Equation (32) agrees with the statement of Theorem 2 for $j=3$.

We proceed in a similar manner for the root nodes 1 and 2. Node 2, for example, needs to update its estimate $\mathbf{z}_{t}^{2^{\natural}}=\mathbb{E}\left(\mathbf{x}_{t}^{2,3,4,5} \mid \mathbf{i}_{t}^{2}\right)$. In order to do so, it needs to estimate the actions of all its descendants. Thus, it needs to estimate the estimates of nodes $1,3,4,5$. Observe that $\mathbb{E}\left(\mathbf{z}_{t}^{1^{\downarrow}} \mid \mathbf{i}_{t}^{2}\right)=0$ because of our assumptions about noise statistics (Assumption A2). Finally, as in the proof of Theorem 1, $\mathbb{E}\left(\mathbf{z}_{t}^{\mathbf{i}^{\mathfrak{\imath}}} \mid \mathbf{i}_{t}^{2}\right)$ for $i=3,4,5$ can be obtained as functions of $\mathbf{z}_{t}^{2^{\downarrow}}$. Combining these observations yields the update equation for $\mathbf{z}_{t}^{2^{\mathfrak{1}}}$. 


\section{Concluding remarks}

In this paper, we described a broad class of decentralized output feedback LQG control problems that admit simple and intuitive sufficient statistics.

In our ongoing work, we take the results of the present paper one step further and derive an explicit and efficiently computable state-space representation for the optimal controller. As with centralized LQG control problems, the optimal estimation and control gains may be computed offline, and the computational complexity is similar as well.

\section{References}

[1] B. Bamieh and P. G. Voulgaris. A convex characterization of distributed control problems in spatially invariant systems with communication constraints. Systems 8 Control Letters, 54(6):575-583, 2005.

[2] V. D. Blondel and J. N. Tsitsiklis. A survey of computational complexity results in systems and control. $A u$ tomatica, 36(9):1249-1274, 2000.

[3] T. H. Cormen, C. E. Leiserson, R. L. Rivest, and C. Stein. Introduction to algorithms, volume 2. MIT press Cambridge, 2001.

[4] Y.-C. Ho and K.-C. Chu. Team decision theory and information structures in optimal control problems-Part I. IEEE Transactions on Automatic Control, 17(1):15-22, 1972 .

[5] J.-H. Kim and S. Lall. Explicit solutions to separable problems in optimal cooperative control. IEEE Transactions on Automatic Control, $\mathrm{PP}(99): 1-1,2014$.

[6] P. Kumar and P. Varaiya. Stochastic Systems: Estimation, Identification and Adaptive Control. Prentice-Hall, 1986 .

[7] B.-Z. Kurtaran and R. Sivan. Linear-quadratic-gaussian control with one-step-delay sharing pattern. Automatic Control, IEEE Transactions on, 19(5):571-574, 1974.

[8] A. Lamperski and J. C. Doyle. Dynamic programming solutions for decentralized state-feedback LQG problems with communication delays. In American Control Conference, pages 6322-6327, 2012.

[9] A. Lamperski and J. C. Doyle. The H2 control problem for quadratically invariant systems with delays. IEEE Transactions on Automatic Control, PP(99):1-1, 2014.

[10] A. Lamperski and L. Lessard. Optimal state-feedback control under sparsity and delay constraints. In IFAC Workshop on Distributed Estimation and Control in Networked Systems, pages 204-209, 2012.

[11] L. Lessard. Decentralized LQG control of systems with a broadcast architecture. In IEEE Conference on Decision and Control, pages 6241-6246, 2012.

[12] L. Lessard and S. Lall. A state-space solution to the twoplayer decentralized optimal control problem. In Allerton Conference on Communication, Control, and Computing, pages 1559-1564, 2011.
[13] L. Lessard and S. Lall. Optimal controller synthesis for the decentralized two-player problem with output feedback. In American Control Conference, pages 6314-6321, 2012.

[14] L. Lessard and S. Lall. Optimal control of two-player systems with output feedback. IEEE Transactions on Automatic Control, PP(99):1-1, 2015.

[15] L. Lessard and A. Nayyar. Structural results and explicit solution for two-player LQG systems on a finite time horizon. In IEEE Conference on Decision and Control, pages 6542-6549, 2013

[16] A. Nayyar and L. Lessard. Optimal control for LQG systems on graphs-Part I: Structural results. arXiv eprint, 2014. http://arxiv.org/abs/1408.2551.

[17] N. Nayyar, D. Kalathil, and R. Jain. Optimal decentralized control in unidirectional one-step delayed sharing pattern. In Allerton Conference on Communication, Control, and Computing, pages 374-380, 2013.

[18] X. Qi, M. Salapaka, P. Voulgaris, and M. Khammash. Structured optimal and robust control with multiple criteria: a convex solution. IEEE Transactions on Automatic Control, 49(10):1623-1640, 2004.

[19] R. Radner. Team decision problems. The Annals of Mathematical Statistics, 33(3):857-881, 1962.

[20] M. Rotkowitz and S. Lall. A characterization of convex problems in decentralized control. IEEE Transactions on Automatic Control, 51(2):274-286, 2006.

[21] N. Sandell, Jr. and M. Athans. Solution of some nonclassical LQG stochastic decision problems. IEEE Transactions on Automatic Control, 19(2):108-116, 1974.

[22] P. Shah and P. Parrilo. $\mathcal{H}_{2}$-optimal decentralized control over posets: A state-space solution for state-feedback. IEEE Transactions on Automatic Control, 58(12):30843096, 2013.

[23] J. Swigart and S. Lall. An explicit state-space solution for a decentralized two-player optimal linear-quadratic regulator. In American Control Conference, pages 63856390, 2010.

[24] J. Swigart and S. Lall. Optimal controller synthesis for a decentralized two-player system with partial output feedback. In American Control Conference, pages 317-323, 2011.

[25] T. Tanaka and P. A. Parrilo. Optimal output feedback architecture for triangular LQG problems. In American Control Conference, pages 5730-5735, 2014.

[26] P. Varaiya and J. Walrand. On delayed sharing patterns. IEEE Transactions on Automatic Control, 23(3):443445, 1978.

[27] H. S. Witsenhausen. A counterexample in stochastic optimum control. SIAM Journal on Control, 6(1):131-147, 1968.

[28] T. Yoshikawa. Dynamic programming approach to decentralized stochastic control problems. In IEEE Conference on Decision and Control, volume 13, pages 185-190, 1974. 